\title{
Application of the microcanonical multifractal formalism to monofractal systems
}

\author{
Oriol Pont, ${ }^{1, *}$ Antonio Turiel, ${ }^{2, \dagger}$ and Conrad J. Pérez-Vicente ${ }^{1, \hbar}$ \\ ${ }^{1}$ Departament de Física Fonamental, Universitat de Barcelona, Diagonal, 647, 08028 Barcelona, Spain \\ ${ }^{2}$ Institut de Ciències del Mar-CSIC, Passeig Marítim de la Barceloneta, 37-49, 08003 Barcelona, Spain
}

(Received 24 February 2006; published 12 December 2006)

\begin{abstract}
The design of appropriate multifractal analysis algorithms, able to correctly characterize the scaling properties of multifractal systems from experimental, discretized data, is a major challenge in the study of such scale invariant systems. In the recent years, a growing interest for the application of the microcanonical formalism has taken place, as it allows a precise localization of the fractal components as well as a statistical characterization of the system. In this paper, we deal with the specific problems arising when systems that are strictly monofractal are analyzed using some standard microcanonical multifractal methods. We discuss the adaptations of these methods needed to give an appropriate treatment of monofractal systems.
\end{abstract}

DOI: 10.1103/PhysRevE.74.061110

PACS number(s): 02.50. $-\mathrm{r}, 05.40 .-\mathrm{a}, 05.10 .-\mathrm{a}, 05.45 . \mathrm{Df}$

\section{INTRODUCTION}

Multicritical and multiscaling systems have become an active research area in nonlinear physics for the last decades. Systems obeying power laws are being reported on a steady basis since a long time ago [1-9]. In this context, the study of critical exponents is a major issue for several reasons. For instance, in a phase transition, there are a large number of relations between the exponents arising from fundamental thermodynamics and statistical mechanics considerations that go beyond any particular system. Close to a critical point, the details on the microscopical dynamics of the system become irrelevant and the macroscopic features are precisely dominated by these exponents.

The paradigm of a scale-invariant, self-similar system is a fractal. The behavior under changes of scale of a physical variable defined on a fractal set usually follows a power law with an exponent related to the fractal dimension $D_{F}$. Therefore, only one parameter, $D_{F}$, is required to characterize the scaling properties of the system. However, there are many examples of complex systems in biology, economy, physics, etc., where this simple description is no longer valid. To characterize them we need a continuous set of exponents, the so-called singularity spectrum. Each one of these exponents - called singularity exponents—gives information about the local degree of regularity or singularity at each point of the system. In general, systems behaving in this way are named multifractals.

There are two main different theoretical formalisms to analyze the scaling properties of fractal and multifractal systems. The classic approach, which we will call canonical formalism (CF), has only a statistical character. The singularity exponents are determined from an averaging process of relevant quantities over different scales. In contrast, the alternative approach, the microcanonical formalism $(\mu \mathrm{CF})$, has a geometric character that allows a characterization of the singularity exponent at each point of the system. The

\footnotetext{
*Electronic address: opont@ub.edu

†Electronic address: turiel@icm.csic.es

*Electronic address: conrad@ffn.ub.es
}

$\mu \mathrm{CF}$ has suffered an important advance in the last years as a consequence of some recent theoretical results [10-12]. It has several advantages that make it particularly interesting with respect to the $\mathrm{CF}$. Thus, $\mu \mathrm{CF}$ analysis requires less statistics (less data points) to achieve a characterization of similar quality [13] and allows a precise geometrical classification of the fractal components of multifractal signals [11]. In addition, by the application of $\mu \mathrm{CF}$ analysis it has been shown that multifractal signals can be reconstructed from the most singular points [12], and eventually $\mu \mathrm{CF}$ methods can be used in forecasting of multifractal time series.

To apply $\mu \mathrm{CF}$ analysis, it is essential to obtain precise estimates of the singularity exponents at each point, as the characterization of a multifractal set is extremely sensitive to biases and errors on them. In practice, when processing multifractal signals from real systems, many limitations on the data, including discretization and sampling, make the task of assessing the correct exponents a challenge.

The previous considerations open some additional interesting issues: There are several methods to perform multifractal analysis based on the $\mu \mathrm{CF}$, but it is not clear how they work when dealing with monofractal systems. These methods are explicitly designed to efficiently assess a continuous spectrum of exponents, but it is not evident whether they are able to collapse this spectrum to a single point. In fact, if one of these methods fails to give the fractal dimension of the signal or it gives a wide spectrum of exponents, then one must be very careful when applying it, since some wrong conclusions could arise from an inappropriate analysis, e.g., to consider that the system is not monofractal but multifractal. In addition, as we will see along this paper, the study of sampled, discretized data representing monofractal systems raises some nontrivial signal processing issues that can affect both $\mathrm{CF}$ and $\mu \mathrm{CF}$ analysis methods. For instance, some adaptations are needed to deal with signals of fractal support. Also an important issue appears when the standard multifractal measure is ill behaved, as it induces a global shift on the singularity exponents. It is important to provide a mechanism able to correct the shift as, in practical situations, one has no prior idea about the scaling features of the analyzed signal. We will consider this problem in detail.

The goal of this paper is hence twofold. First, to study the validity of the $\mu \mathrm{CF}$ for the analysis of monofractal systems; 
we will use different $\mathrm{CF}$ and $\mu \mathrm{CF}$ analysis methods and discuss their reliability, advantages, and limitations-in particular, in terms of their ability to obtain a single point or very narrow range of points in the singularity spectra. Second, to present a discussion on the adaptations to deal with signals defined on fractal supports and on the generalization of multifractal measures when the standard definition is illbehaved.

This paper is organized as follows: In the next section, we briefly introduce several standard methods of multifractal analysis based on the $\mu \mathrm{CF}$. In Sec. III we introduce two analysis functionals, namely the linear increment and the measure. Also, we discuss some generic adaptations needed by the methods to correctly analyze the signals. Section IV is devoted to the introduction of the monofractals analyzed in this paper, while in Sec. V we show the obtained results. Finally, in Sec. VI we present our conclusions.

\section{MULTIFRACTAL METHODS BASED ON THE $\mu$ CF}

The $\mu \mathrm{CF}$ allows one to characterize the local scaling features of multifractal signals. In order to unveil this local structure, singularity analysis methods are applied. Given a signal $s(t)$, singularity analysis consists of assigning to each point $t$ a singularity exponent $h(t)[11,14]$. We will denote the singularity manifold $F_{h}$ as the set of points sharing the same value of singularity,

$$
F_{h} \equiv\{t: h(t)=h\} .
$$

The singularity manifolds $F_{h}$ are sets of very irregular nature, and they typically are of fractal character, with nontrivial fractal dimensions. For that reason, the singularity manifolds $F_{h}$ are also usually known as fractal components. We will denote by $D(h)$ the fractal dimension of the manifold $F_{h}$. When $D(h)$ is regarded as a function of $h$ it is called the singularity spectrum of the signal.

The signal $s(t)$ is analyzed by means of an appropriate operator $\mathbb{T}_{r}$ that acts on $s$ and returns a new function $\mathbb{T}_{r} s(t)$. The operator depends on a scale variable $r$, which characterizes the locality of its actuation. To assign to each point $t$ a singularity exponent $h(t)$, the following relation must hold:

$$
\mathbb{T}_{r} s(t)=\alpha_{\mathrm{T}}(t) r^{h(t)}+o\left(r^{h(t)}\right) .
$$

We will say that the signal $s(t)$ is multifractal in the $\mu \mathrm{CF}$ sense if there exists a functional $\mathbb{T}_{r}$ such that Eq. (2) is verified and the obtained singularity spectrum $D(h)$ is a convex curve, namely, it has negative second derivative for all $h$. This second condition is a consequence of Jensen's inequality [15], meaning that the distribution of $T_{r} s$ can be directly connected with the existence of an infinitely divisible multiplicative cascade [16]. In fact, the singularity spectrum completely characterizes the statistics of the multiplicative process [16-19]. Also, we can obtain the singularity spectrum from the statistics of the multiplicative process, so that we can establish a bidirectional connection between statistical and geometrical self-similarity.

The implementation of $\mu \mathrm{CF}$ analysis can be done following different strategies. Each one has its own difficulties and advantages when they are used in the analysis of monofractal systems. In our work, we have considered two $\mu \mathrm{CF}$ methods: the local singularity method and the punctual singularity method. We have also included the moment method, which is proper to the $\mathrm{CF}$, to highlight the differences between geometric and statistical characterization. A CF method can be applied in the context of $\mu \mathrm{CF}$ because a system which is multifractal in the $\mu \mathrm{CF}$ is also multifractal according to the CF [19]. In the following, we will briefly see how to apply these methods to obtain the singularity spectrum but, for a more detailed revision, we refer the reader to Ref. [13].

The main point common to all these methods is to determine the probability $\rho_{r}(h)$ of observing a singularity $h$ at scale $r$, something that is completely determined by the fractal dimension $D(h)$ of the associated fractal component $F_{h}$ [20], namely,

$$
\rho_{r}(h) \propto r^{d-D(h)},
$$

where $d$ is the topological dimension of the embedding space of the signal. Fractal sets cannot have a dimension higher than that of their embedding, so $D(h) \leqslant d \forall h$. In this paper, we assume $d=1$ although all of the following methods can be straightforwardly extended to analyze $d$-dimensional signals.

\section{A. Local singularity method}

In this method, we obtain an estimate of the singularity exponent $h(t)$ at any point $t$ through a log-log linear regression of Eq. (2) in a range of scales. Then, the empirical distribution $\rho_{r_{0}}(h)$ is estimated from the histogram of experimental values of $h$ at the resolution scale $r_{0}$. In fact, any scale can be used but, at the resolution scale $r_{0}$, we have more points to evaluate the histogram. Taking Eq. (3) into account, we can derive the singularity spectrum, namely,

$$
D(h)=d-\frac{\ln \left[\rho_{r_{0}}(h) / \rho_{r_{0}}\left(h_{1}\right)\right]}{\ln r_{0}},
$$

where $\rho_{r_{0}}\left(h_{1}\right)$ is an appropriate constant that allows us to remove the implicit normalization factor in Eq. (3). To determine this constant, we require that $D\left(h_{1}\right)=d$ so that $\rho_{r}\left(h_{1}\right)$ does not depend on the scale and allows us to normalize the distribution $\rho_{r}(h)$. Notice that if such singularity $h_{1}$ exists, it is necessarily the modal point (i.e., the maximum of the singularity distribution), as no fractal component $F_{h}$ can have a dimension $D(h)$ higher than $d$. Therefore, the normalization becomes trivial: we normalize the histogram by its maximum. The question is if such a maximum has actually associated dimension $D\left(h_{1}\right)=d$. For signals with total support, there is at least one fractal manifold $F_{h_{1}}$ that attains the maximum dimensionality, i.e., $D\left(h_{1}\right)=d$ [21]. For signals having strictly fractal support, i.e., a support with fractal dimension $D_{\text {supp }}<d$, we can analyze the whole real domain of $t$ and formally assign the value $h_{1}(t)=\infty$ to all the points outside the support [which means that $s(t)=$ constant on those points]. Then $F_{h_{1}}$ is a total support manifold with associated dimension $D\left(h_{1}\right)=d$ and the proposed normalization also works. 


\section{B. Punctual singularity method}

This method is an extreme simplification of the local singularity method: singularities are estimated in a very crude fashion, from the behavior of the operator at the minimum scale $r_{0}$ instead of a linear regression. Namely, we assume that $r_{0}$ is so small that $\ln \alpha_{\mathrm{T}}$ in Eq. (2) is negligible in comparison with $\ln r_{0}$. We obtain the punctual singularity exponent estimate, $\tilde{h}(t)$, given by

$$
\tilde{h}(t) \equiv \frac{\ln \mathbb{T}_{r_{0}} s(t)}{\ln r_{0}} .
$$

As a matter of fact, for many real signals with good enough resolution, $\tilde{h}(t)$ is a good estimate of $h(t)$ [13]. Then, we proceed as in the previous method: we compute the empirical distribution of $\tilde{h}$ and from it we apply the histogram formula given in Eq. (4) to obtain the singularity spectrum $D(h)$.

Both the local singularity method and the punctual singularity method obtain the singularity spectrum from the histogram of singularity exponents. This fact allows us to estimate the error bars of the spectrum, by considering the sampling error associated to each bin of the histogram. If we consider an uncertainty of three standard deviations (about $99 \%$ of confidence), the error bars of the singularity spectrum are given by

$$
\Delta D(h)=\frac{3 \sqrt{N_{h}}}{\ln r_{0}},
$$

where $N_{h}$ is the number of events in the bin that corresponds to the singularity exponent $h$. The reader can find a step-bystep proof of the previous formula in Ref. [13].

\section{Moment method}

This is the classical method, based on the $\mathrm{CF}$ $[16,18,22-25]$, but also applicable under the $\mu \mathrm{CF}$ framework. Parisi and Frisch's formula [19] establishes a link between the global, statistical exponents associated to the $\mathrm{CF}$ approach and the local, geometrical exponents associated to $\mu \mathrm{CF}$. In the following lines we schematically reproduce the Parisi and Frisch proof which renders this link explicit.

When computing the order $p$ moments of the variable $\mathbb{T}_{r} s$ we have

$$
\left\langle\left|\mathbb{T}_{r} S\right|^{p}\right\rangle \propto \int d h r^{d-D(h)} r^{p h} .
$$

When $r$ is small enough, the integral will be dominated by the minimum value of the exponent $[d-D(h)+p h]$, so we obtain that

$$
\left\langle\left|\mathbb{T}_{r} s\right|^{p}\right\rangle \propto r^{\tau_{p}}, \quad r \ll 1,
$$

where

$$
\tau_{p}=\inf _{h}[p h+d-D(h)]
$$

that is, the canonical exponents $\tau_{p}$ are given by the Legendre transform of the singularity spectrum $D(h)$. Equation (9) is the famous Parisi and Frisch formula [19]. If the singularity spectrum is convex, we can invert the Legendre transform and express the singularity spectrum as a function of the canonical exponents,

$$
D(h)=\inf _{p}\left(p h+d-\tau_{p}\right) .
$$

For the validity of this method, we need to make an explicit appeal to the convexity of the function $D(h)$. As we require that signals in the $\mu \mathrm{CF}$ possess convex spectra, this limitation is not constraining our study.

\section{FUNCTIONALS}

Different choices for the functional $T_{r} s$ are possible. Similar functionals usually lead to the same singularity characterization $h(t)$ of the signal, but sometimes not all of them lead to exactly the same results. In the next paragraphs, we describe the functionals used in our work.

\section{A. Linear increment}

A commonly used functional is the linear increment, $\left|\Delta_{r} s\right|$, given by

$$
\mathbb{T}_{r} s(t)=\left|\Delta_{r} s\right|(t) \equiv|s(t+r)-s(t)| .
$$

This functional has evidenced the microcanonical multifractal scaling in the sense of Eq. (2) when applied to several real world systems $[17,24]$. However, this is true only when the signal under analysis belongs to the very restrictive class of multiaffine functions [22], which are a subset of microcanonical multifractals. A classical example of experimentally observed multiaffine function is given by field velocities recorded in flows under fully developed turbulence [17]. The main advantage of multiaffine functions is the closeness to classical concepts in functional analysis as Hölder exponents; in fact, a multiaffine function $s(t)$ with singularity exponents $h(t)$ has a Hölder exponent at each point $t$ that coincides with $h(t)$ so, from Eqs. (2) and (11), it verifies that

$$
|s(t+r)-s(t)| \sim r^{h(t)},
$$

where the symbol $\sim$ means asymptotically proportional to, as $r \rightarrow 0$, so that Eq. (12) has a meaning equivalent to Eq. (2), but in a simplified notation.

In practice, multiaffinity is often impossible to assess due to the presence of long-range correlations which mask large exponents $[21,26]$. Nevertheless, in some cases, it is still possible to extend the class of multiaffine functions using a wavelet-based functional which generalizes the linear increment, namely,

$$
\mathbb{T}_{r} s(t)=T_{\Psi} s(t, r) \equiv \int d t^{\prime} s\left(t^{\prime}\right) \frac{1}{r^{d}} \Psi\left(\frac{t^{\prime}-t}{r}\right),
$$

where the symbol $T_{\Psi} s(t, r)$ stands for the classical definition $[27,28]$ of the wavelet projection of the signal $s$ on the wavelet $\Psi$ at the point $t$ and scale $r$. The wavelet $\Psi$ must be required to have zero mean, so that it consists (at least) of a positive and a negative part, and hence it realizes a weighted difference, generalizing that of the linear increment and filtering the effects of long-range correlations [21]. Wavelet- 
based multiaffine analysis has allowed one to characterize the multifractal structure of many different systems $[21,26,29-32]$, although only in the context of the CF.

\section{B. Multifractal measure}

Another possibility to attain a geometric microcanonical characterization comes from the use of multifractal measures $[23,24]$. A measure $\mu$ is a $\sigma$-additive function operating on sets $A$ such that it assigns a positive value to each set, $\mu(A)>0 \forall A$. A measure that has been reported to unveil $\mu \mathrm{CF}$ multifractal properties for many different signals $[11,33,34]$ is the gradient modulus measure, which is given by

$$
\mu(A)=\int_{A} d t^{\prime}|\nabla s|\left(t^{\prime}\right)
$$

for any set $A$. This definition allows one to define a measure functional,

$$
\mathbb{T}_{r} s(t)=\frac{1}{r^{d}} \mu_{r}(t) \equiv \frac{1}{r^{d}} \int_{B_{r}} d t^{\prime}|\nabla s|\left(t^{\prime}\right),
$$

where $B_{r}(t)$ is the ball of radius $r$ and centered on $t$, and $\mu_{r}(t)$ stands for the measure of that ball, $\mu\left[B_{r}(t)\right]$. Again, in order to filter long-range correlations and provide a smooth interpolation scheme, it is convenient to generalize the definition of the functional above using wavelet projections of the measure $[11,35,36]$, in a similar way to what was done before with linear increments,

$$
\mathbb{T}_{r} s(t)=T_{\Psi}|\nabla s|(t, r) \equiv \int_{-\infty}^{\infty} d t^{\prime} \frac{1}{r^{d}} \Psi\left(\frac{t^{\prime}-t}{r}\right)|\nabla s|\left(t^{\prime}\right) .
$$

Contrary to the wavelet-based generalization of linear increments, here the wavelet $\Psi$ is not required to have zero mean and positive functions can be used [11]. Wavelet projections of multifractal measures have allowed one to unveil $\mu \mathrm{CF}$ multifractality when applied to systems as diverse as natural images [11], econometric time series [37], meteorological images [38], and oceanographical satellite images [34].

The reader can notice that the scaling dependence on $r$ for the measure definitions of $T_{r}$, Eqs. (15) and (16), will differ in a factor $r$ from the linear increment definitions of $\mathbb{T}_{r}$, Eqs. (11) and (13), due to the fact that the analyzed quantity in measures is the gradient of the signal, while in linear increments it is the signal itself. Hence, a shift on +1 should be expected in the estimation of the singularity exponents $h(t)$ $[11,31]$.

A good criterion to use a functional or another is to determine the quality of a regression among several scales in Eq. (2). In the following, we will deal with these two kinds of functionals that we have presented, namely the linear increment, Eq. (11), and the measure functional, Eq. (15); and also with their wavelet extensions, Eqs. (13) and (16), respectively. These are the most commonly used functionals and have been shown to work in many practical situations. We will see in each instance the appropriateness of each functional according to the signal to be analyzed and the adaptations needed.

\section{Wavelet processing of fractal sets with nontotal support}

As discussed, wavelet projections were introduced to filter noise and long-range correlations. However, they introduce some spatial dispersion which may be a drawback when analyzing monofractals with fractal support. The wavelet projections of points close to any support point will have a small but nonzero contribution, and so the point could be erroneously assigned a large (but not infinity, as it should be) value of singularity exponent. Nevertheless, this problem can be simply circumvented if we isolate the support points and then restrict the study of wavelet projections to these points. Extracting the support is a simple task: zero-gradient points are outside the support, all the other points belonging to it (mathematically speaking, the support is the topological closing of the set of non-null gradients but, over discretized signals, taking the closing is something more formal than effectively real). When numerically separating support and nonsupport points, a small but finite threshold must be used to decide if the gradient is negligible. It may happen that some points in the support with very small gradient are incorrectly considered as nonsupport points but, in practice, the observed proportion of incorrectly classified points is very low and does not affect the final result.

\section{Generalization of the measure for ill-behaved gradients}

A standard finite-difference discretization at resolution scale $\delta$ of the multifractal measure defined in Eq. (15) is the following:

$$
\mu_{r}^{(\delta)}(t)=\sum_{i=1}^{N} \delta\left|\nabla^{(\delta)} s(t+i \delta)\right|,
$$

where $N=r / \delta$ is the effective number of points that contribute to the measure. The gradient, $\nabla s(t)$, is approximated at resolution $\delta$ as

$$
\nabla^{(\delta)} s(t)=\frac{\Delta_{\delta} s(t)}{\delta},
$$

where $\Delta_{\delta} s(t)=s(t+\delta)-s(t)$.

For well-behaved gradients, all the dependency on $\delta$ is removed when limits are taken, i.e.,

$$
\nabla^{(\delta)} s(t) \underset{\delta \rightarrow 0}{\rightarrow} \nabla s(t)
$$

and $\mu_{r}^{(\delta)}$ will converge to the actual measure $\mu_{r}$ as $\delta \rightarrow 0$. However, this convergence requires that the gradient $\nabla s$ is well defined, at least in a distributional sense. That is, $\nabla s$ can diverge to $\infty$ at some points, but it must follow a well-defined distribution. In other words, the curve $|\nabla s|(t)$ can diverge in a zero-measure set of points, but it must be integrable.

Consider two measures defined on the same interval, approximated with two different scale quanta $\delta, \delta^{\prime}$. If $\nabla s$ is well defined, both converge to the same quantity, namely,

$$
\mu_{r}^{(\delta)}(t)=\mu_{r}^{\left(\delta^{\prime}\right)}(t), \quad \delta, \delta^{\prime} \rightarrow 0
$$

and both approximate $\mu_{r}(t)$. 
However, when the gradient $\nabla s$ is not well defined, its discrete estimation $\nabla^{(\delta)} s$ does depend on $\delta$. This is typically the case of fractal functions with infinite curve length, i.e., infinite $\mu_{r}$. If $\nabla s$ is not defined in a distributional sense, neither is $\mu_{r}$, and the numerical, finite-size estimates of the measure at a given point $t, \mu_{r}^{(\delta)}(t)$ and $\mu_{r}^{\left(\delta^{\prime}\right)}(t)$, cannot converge to a fixed quantity,

$$
\mu_{r}^{(\delta)}(t) \neq \mu_{r}^{\left(\delta^{\prime}\right)}(t), \quad \delta, \delta^{\prime} \rightarrow 0 .
$$

Therefore, $\mu_{r}^{(\delta \rightarrow 0)}(t)$ will not scale as $r^{1+h(t)}$ as expected. On the contrary, it usually grows proportionally to the number of points $N$ (bulk scaling), leading to an incorrect estimation of singularity exponents, $h_{\mathrm{est}}(t)=0$.

To solve this problem, one option would be to scale the infinitesimal $\delta$ according to $r$, i.e., $\delta=r / N$, thus leading to the right scaling in $r, \mu_{r}^{(\delta)}(t) \sim r^{1+h(t)}$. However, the signals to be analyzed are sampled at a fixed resolution scale $\delta$ and we cannot change it.

We can proceed in a different way, providing a redefinition of the measure, well behaved and giving access to the true scaling properties of the signal. In order to give a physical meaning to the measure, we must require that $\mu_{r}^{(\delta)}$ $=\mu_{r}^{\left(\delta^{\prime}\right)}$ as $\delta, \delta^{\prime} \rightarrow 0$. So, we propose to regularize the measure in the following way:

$$
\mu_{r} \equiv \lim _{\delta \rightarrow 0} \frac{\mu_{r}^{(\delta)}}{N^{\alpha}}, \quad N=\frac{r}{\delta},
$$

where $\alpha$ is an exponent to regularize the behavior of $\mu_{r}$. The value of $\alpha$ is not known a priori, but can be easily estimated from two different discretizations, $\delta$ and $\delta^{\prime}$, which are assumed to be very small,

$$
\frac{\mu_{r}^{(\delta)}}{N^{\alpha}}=\frac{\mu_{r}^{\left(\delta^{\prime}\right)}}{N^{\prime \alpha}}
$$

with $r=N \delta=N^{\prime} \delta^{\prime}$. The expected value of $\mu_{r}^{(\delta)}$ is

$$
\left\langle\mu_{r}^{(\delta)}\right\rangle=N^{1+h_{\text {est }}}\left\langle\mu_{\delta}^{(\delta)}\right\rangle,
$$

where $h_{\text {est }}$ is the incorrectly estimated singularity exponent. From Eq. (17), it follows that $\left\langle\mu_{\delta}^{(\delta)}\right\rangle=\left\langle\left|\Delta_{\delta} s\right|\right\rangle$ and taking expected values on Eq. (23) we obtain that

$$
\delta^{\alpha-h_{\mathrm{est}}-1}\left\langle\left|\Delta_{\delta} s\right|\right\rangle=\delta^{\alpha-h_{\mathrm{est}}{ }^{-1}}\left\langle\left|\Delta_{\delta^{\prime}} s\right|\right\rangle
$$

so we can easily estimate $\alpha$ from

$$
\alpha=1+h_{\mathrm{est}}-\frac{\ln \left(\left\langle\left|\Delta_{\delta^{\prime}} s\right|\right\rangle /\left\langle\left|\Delta_{\delta} s\right|\right\rangle\right)}{\ln \left(\delta^{\prime} / \delta\right)} .
$$

Notice that we can always apply the generalized measure definition, Eq. (22), that is, we do not need to know a priori whether the signal $s$ has an undefined gradient or not. If the standard measure is well behaved $\mu_{r}^{(\delta)}$ and $\mu_{r}^{\left(\delta^{\prime}\right)}$ directly approximate $\mu_{r}$ and, from Eq. (23), it follows that $\alpha=0$ and the correct singularity exponent $h_{\text {est }}=h$ is directly estimated (notice that $h$ is the singularity exponent referred to the gradient, not to the signal). Therefore, we will always make use of the generalized definition, Eq. (22).

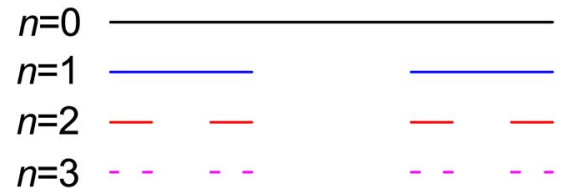

FIG. 1. (Color online) Iterative steps of generation of a $(1,0,1)$ Cantor set.

\section{MONOFRACTAL DATASETS}

We will deal with two different types of synthetic monofractal signal, commonly referred to in the literature. The first type is the $\vec{p}$ order $Q$-adic Devil's staircase ( $\vec{p} \mathrm{DS}$ ); the second one is the fractional Brownian motion (fBm). We have chosen these because they are very simple signals but have enough richness and complexity to lead to interesting processing issues.

\section{A. Definition of $\vec{p}$ DS}

Signals of this type [1] are generated by integrating the uniform $\vec{p}$ Cantor measure $K_{\vec{p}}$ in the interval $[0,1]$. Let us define the notation first. $Q$ will be an integer number $Q \in \mathbb{N}$. The vector $\vec{p}$ is a $Q$-dimensional vector for which each component can only take the value 0 or $1, \vec{p} \in Z_{2}^{Q}$. We define by $p$ the norm-1 of $\vec{p}$, that is, the total number of 1 's in $\vec{p}$. We will define the measure $K_{\vec{p}}$ as the limit of the sequence of measures $K_{\vec{p}}^{n}$, which are successive approximations to the final measure, namely,

$$
K_{\vec{p}}=\lim _{n \rightarrow \infty} K_{\vec{p}}^{n} .
$$

The measures $K_{\vec{p}}^{n}$ are obtained by successive subdivision of each subinterval in new $Q$ subpieces, from which $Q-p$ are removed and $p$ kept. In a more formal way we have

$$
K_{\vec{p}}^{n}=\frac{Q^{n}}{p^{n}} \sum_{i_{1}, i_{2}, \ldots, i_{n}=1}^{Q} p_{i_{1}} p_{i_{2}} \ldots p_{i_{n}} \chi_{\left[0 . i_{1} i_{2} \ldots i_{n}, 0 . i_{1} i_{2} \ldots\left(i_{n}+1\right)\right]}
$$

where $\chi_{A}$ is the set measure associated to the set $A, \chi_{A}(B)$ $=|A \cap B|$. The limit measure $K_{\vec{p}}$ is then a unitary measure with support on the $\vec{p}$ Cantor set, namely the set such that the $Q$-adic representation of its points does only contain the digits corresponding to the non-null coordinates of $\vec{p}$. A visual realization of this generating process is presented in Fig. 1.

In addition to the classical, deterministic definition of the $\vec{p} Q$-adic Cantor measure $K_{\vec{p}}$ given above, we can also define the random $p Q$-adic Cantor measure $R_{p ; Q}$, just by choosing the $p$ non-null components of the vector $\vec{p}$ at random at each step.

The $\vec{p}$ Devil's staircase ( $\vec{p}$ DS) is the continuous function $s(t), t \in[0,1]$, defined as the $\vec{p}$ Cantor measure of the interval $[0, t]$,

$$
s(t) \equiv K_{\vec{p}}([0, t]) .
$$

We have an analogous expression for the random $p Q$-adic DS, obtained by integrating the random $p Q$-adic Cantor measure. 

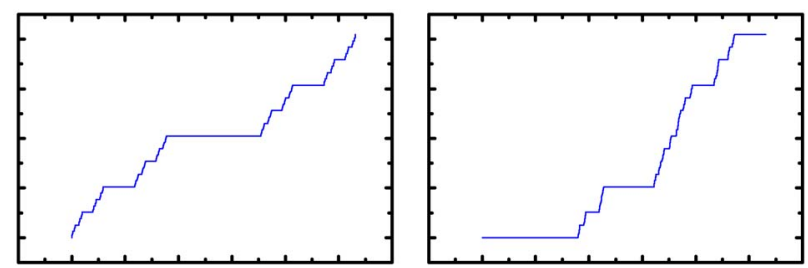

FIG. 2. (Color online) Deterministic $(1,0,1)$ DS (left-hand side) and random $p=23$-adic DS (right-hand side).

DS have some relevant features, if $0<p<Q$, it is nondecreasing, $s(0)=0, s(1)=1$, and it has zero derivative in all the points but a zero-measure set. The trivial cases $p=0$ $[\Rightarrow s(t)=0]$ and $p=Q[\Rightarrow s(t)=t]$ are degenerated, so we will ignore them. In Fig. 2 we show a deterministic and a random DS.

Each point in a DS can be associated with a singularity exponent, depending on whether the point belongs to the support of the measure or not. Let $S_{\vec{p}}$ be the support of the Cantor measure, $S_{\vec{p}}=\operatorname{supp}\left(K_{\vec{p}}\right)$; hence, if $t$ belongs to the support, $t \in S_{\vec{p}}$, we have that

$$
|s(t+r)-s(t)| \sim r^{D_{\text {supp }}} \quad \forall r
$$

with a Hölder exponent $D_{\text {supp }}=\ln p / \ln Q$ that coincides with the Hausdorff dimension of the support $S_{\vec{p}}$ [20]. On the contrary, if $t \notin S_{\vec{p}}$, we have that there is a finite $r_{0}>0$ such that

$$
|s(t+r)-s(t)|=0 \quad \forall r<r_{0}
$$

so the Hölder exponent associated to these points is $\infty$.

\section{B. Definition of the $\mathrm{fBm}$}

Fractional Brownian motions (fBm's) are natural extensions to Brownian motions. They are stationary continuous random processes with Gaussian marginal distributions and power law correlations. Namely, $s(t)$ is a fBm of index $H$ if

(i) with probability $1, s(0)=0$ and $s(t)$ is a continuous function of $t$.

(ii) $\forall t \geqslant 0$ and $\forall r>0, s(t+r)-s(t)$ is Gaussianly distributed, with mean 0 and dispersion $r^{H}$.

The $\mathrm{fBm}$ generalizes the classical concept of Brownian motion to the cases in which disjoint increments are positively or negatively correlated. The index value $H=1 / 2$ is associated to the classical uncorrelated Brownian motion, while $H>1 / 2$ represents processes in which increments are positively correlated, and $H<1 / 2$ leads to processes in which increments are negatively correlated. In Fig. 3 we show examples of negatively correlated, uncorrelated, and positively correlated fBm's.

Each point in a $\mathrm{fBm}$ can be associated with the same Hölder exponent, which coincides with $H$ [20]; this follows from the condition (ii) in the definition above. Hence, we have that

$$
|s(t+r)-s(t)| \sim r^{H} .
$$

This result is also required, as a strictly necessary requirement, from the continuity condition (i). Namely, the continuity allows one to subsplit the interval $(t, t+r)$ as $n$ subintervals, each one contributing a difference to the global difference, all random but all with dispersion $(r / n)^{H}$. The scaling $r^{H}$ is a result of a thermalization when $n \rightarrow \infty$. For that reason, when dealing with discretized data with a finite number of possible subdivisions, deviations from the central value $H$ should be expected.

In our work, we have generated $\mathrm{fBm}$ signals using the Wood-Chan algorithm [39] which is an exact generation method. By "exact" we mean that the increments, Eq. (32), are exactly Gaussianly distributed, even at the resolution scale (that is, increments at any level follow a Gaussian distribution, not a distribution which converges to a Gaussian just as a limit behavior). In addition, the Wood-Chan method is not greatly affected by numerical instabilities, something that plagues other generating methods, especially when used to generate negatively correlated signals.

In the case of $\mathrm{fBm}$, the gradient $\nabla s$ is not well defined. The discrete estimate of the gradient at scale $\delta, \nabla^{(\delta)} s$, as defined in Eq. (18), has a normal distribution with mean 0 and dispersion $\delta^{H-1}$, which becomes undefined when $\delta \rightarrow 0$ (as $H<1$, it has infinite zero-order moment). Therefore, to avoid wrongly estimated scalings in the analysis with measure functionals, we must use the generalized definition of the measure presented in Sec. III D. In fact, the naive definition of the measure leads to an incorrect scaling $\mu_{r} \sim r^{1}$ instead of $\mu_{r} \sim r^{H}$ as expected. We give a theoretical proof of this wrongly estimated scaling in the Appendix.

\section{RESULTS}

We have used the three methods presented in Sec. II and the two kinds of functional $\mathrm{T}_{r}$ of Sec. III, namely the linear increment and the measure (also their wavelet-based equivalents, when needed), to analyze several ensembles of Devil's staircases and fractional Brownian motions. The waveletbased definitions of the functionals allow us to filter noise and avoid local fluctuations in the estimation of $h(t)$. In the local singularity method we require the best possible estimate of $h(t)$ at each $t$ so that, to decrease the influence of noise and numerical instabilities, the wavelet-based definitions, Eqs. (13) and (16), should be used. We use the first derivative of the Gaussian as an analysis wavelet for the generalized linear increment and a simple Gaussian for the measure functional.
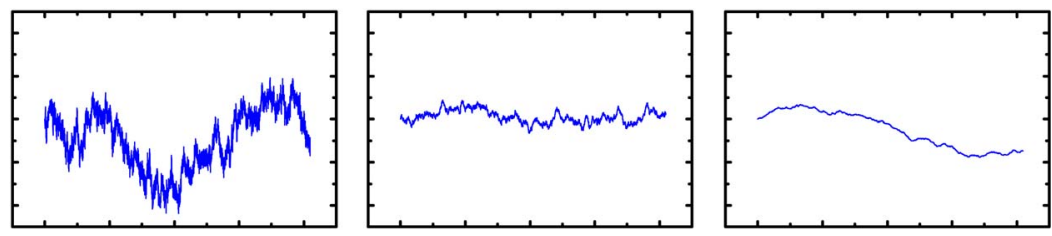

FIG. 3. (Color online) fBm with $H=1 / 4$ (lefthand side), $H=1 / 2$ (center), $H=3 / 4$ (right-hand side). 

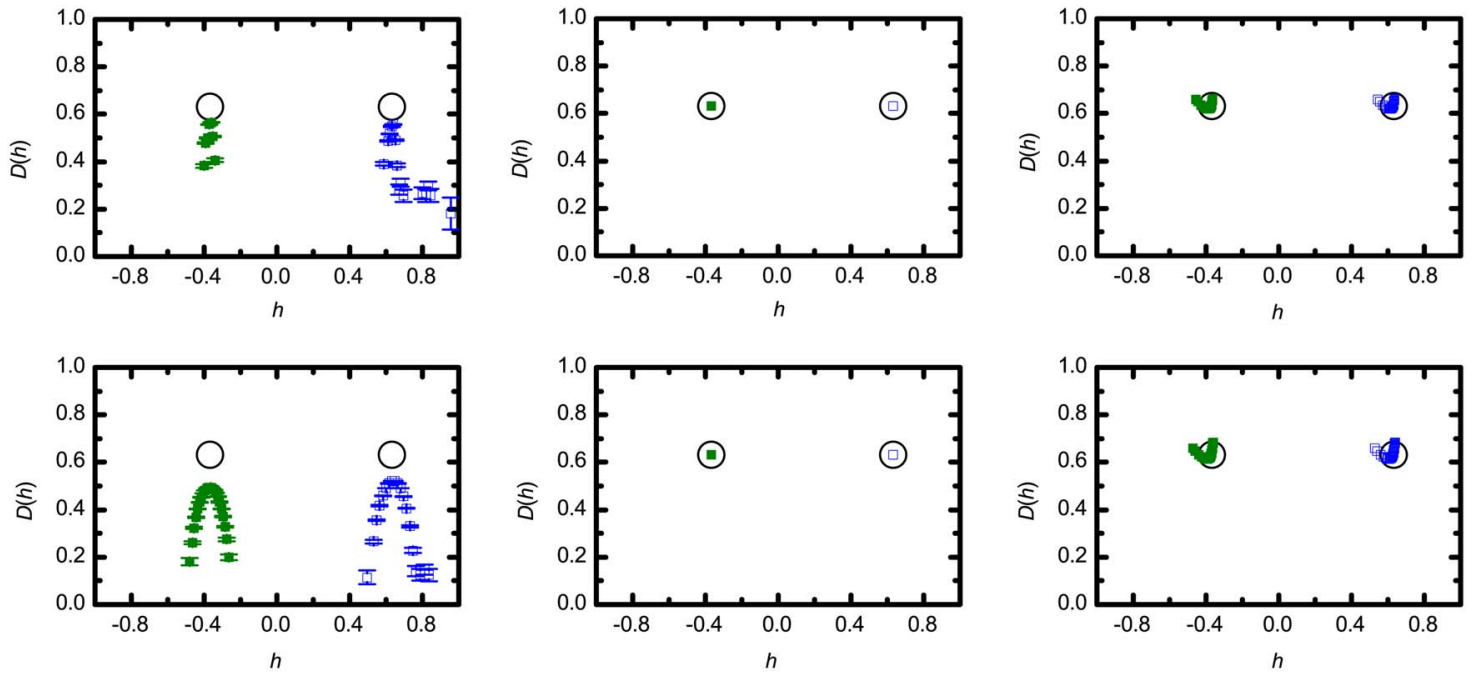

FIG. 4. (Color online) Estimated singularity spectra $D(h)$ computed with the local singularity method (left-hand side), the punctual singularity method (center), and the moment method (right-hand side), for the (1,0,1) DS (first row) and the random $p=2$ 3-adic DS (second row). The spectra are estimated with linear increments $(\square)$ and multifractal measures $(\boldsymbol{\square})$. The circle indicates the theoretical singularity exponent and associated Hausdorff dimension.

For the punctual singularity method, we analyze only at the scale of resolution $r_{0}$ with the simple definitions; the use of wavelets has almost no effect, as they extend over a very small scale, and hence the wavelet projections give almost the same result as the nonwavelet functional from where they are derived. In addition, linear increments and measures are essentially identical when this method is applied to discretized data: the gradient becomes a finite difference of scale $r_{0}$ (i.e., identical to a linear increment) divided by the scale step $r_{0}$, so that both functionals give exactly the same estimates of the exponents, up to the factor +1 discussed in Sec. III, which is due to the presence of the $1 / r_{0}$ factor in the definition of the gradient. Finally, regarding the moment method, wavelets are unneeded, as we only need a statistical determination of the singularity exponents, not a precise estimate at each point. Therefore, we make use of the simple definitions, Eqs. (11) and (15).

In the local singularity method and the moment method, a scale range from $128 r_{0}$ to $32768 r_{0}$ exponentially sampled has been used. The smallest scales have not been analyzed to avoid discretization effects.

We include the error bars of the spectra of the microcanonical methods, using the formula (6). Notice that these error bars express only the statistical error, but do not account for systematic deviations, whose nature is discussed later on.

\section{A. Study of the $\vec{p}$ DS}

For the experiences concerning the Devil's staircases, we have generated two different ensembles. The first one consists of a single deterministic $(1,0,1)$ DS. The second one consists of 10 different random $p=23$-adic DS. In both cases we have generated series of exactly 14348907 points ( $n=15$ scales of generation). Figure 4 shows the spectra obtained for each method and functional.

\section{Local singularity method}

As discussed in Sec. III C, we restrict the wavelet projections to the numerically determined support of the $\vec{p}$ DS. However, estimated singularity exponents are affected by some dispersion. The singularity spectra peak around the correct value with a relatively small dispersion, a bit greater for random DS than for deterministic DS, for both linear increment and measure functionals. The dimension at the peak is slightly smaller than its theoretical value $D$, the fractal dimension of the DS, because dispersion in the singularity exponents splits the fractal support into various submanifolds, all of less fractal dimension than the support. When we consider the manifold formed by the union of all these subsets (by taking a coarser binning in the histogram of singularities) we indeed recover the actual dimension $D$.

\section{Punctual singularity method}

In this case, this method attains the best performance, as it achieves almost perfect estimations of the singularity spectra for both deterministic and random Devil's staircases. This is due to the fact that, at resolution level, $r_{0}$, the operator $\mathbb{T}_{r} s$ only takes two values, 1 and 0 . The set of nonzero points coincides exactly with the support. This is a bit unphysical situation, but certainly advantageous in this case.

\section{Moment method}

When the moment method is applied to a generic multifractal signal, the range of moments used in the analysis must include values of $p$ large enough to ensure that the tails of the singularity spectrum $D(h)$ are retrieved. According to Eq. (9), large-order, negative moments correspond to the right tail of $D(h)$ and large-order, positive moments to the left tail, due to the convexity of the $\tau_{p}$ vs $p$ curve discussed in Sec. II C. For monofractals, the spectrum consists of a single point and the curve $\tau_{p}$ is always a straight line. For the 


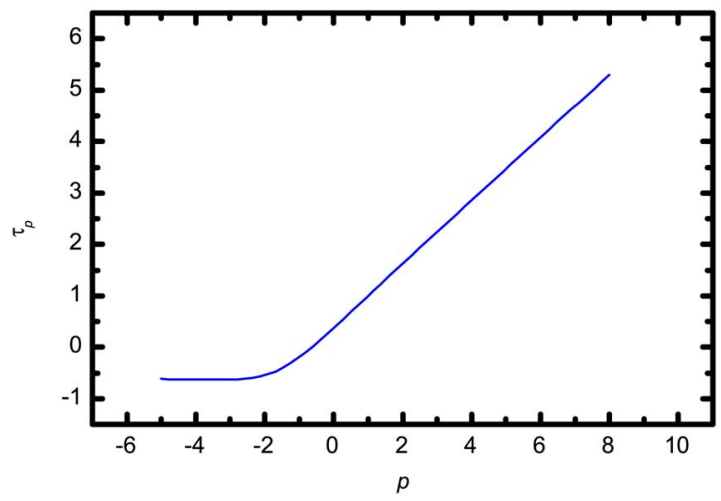

FIG. 5. (Color online) $\tau_{p}$ computed with the moment method for the $(1,0,1)$ DS.

particular case of DS, the slope of the curve $\tau_{p}$ is $D$ for linear increments, Eq. (11), and $D-1$ for measures, Eq. (15).

Experimental $\tau_{p}$, either using linear increments or measures, exhibit a loss of convexity at negative, large-order moments. For any multifractal system the curve $\tau_{p}$ vs $p$ must be convex, as this is a direct consequence of Jensen's inequality (see discussion in Sec. II). Therefore, a concave zone must be interpreted as a numerical artifact due to loss of accuracy and hence we need to truncate the curve $\tau_{p}$ at least at the inflection point $p_{i}$. The value of $p_{i}$ depends on the size of the generated signal, although $p_{i}$ increases in absolute value at a very slow fashion with the total number of points in the series. An example of resulting $\tau_{p}$ curve, shown in Fig. 5 , indicates that it seems appropriate to stop at order -1 to avoid the artificial concavity zone, even if that restricts the analysis to the central part of the singularity spectrum. Therefore, the range from $p=-1$ to $p=8$ has been analyzed.

The estimated spectra are pointlike, with a small dispersion for both linear increment and measure functionals. Although dispersion is equal to or less than in the local singularity method, the spectra do not seem to follow well-defined curves. The peak of the $D(h)$ curves is very difficult to localize, so neither the singularity exponent nor the fractal dimension can be precisely estimated.

\section{B. Study of the fBm}

For this study, we have generated three ensembles of fractional Brownian motions, each one associated to exponents $H=1 / 4, H=1 / 2$, and $H=3 / 4$. The three ensembles consist of 10 series, of 16777216 points each, generated through the Wood-Chan algorithm. Figure 6 shows the spectra obtained for each method and functional.

Although theory predicts that increments of the continuous fBm depend on the scale parameter $r$ as a power law of Hölder exponent $H$ for any point in the series, as shown in Eq. (32), this result is a consequence of the continuity and hence, when dealing with discretized signals, finite resolution effects are relevant. For discretely generated series, linear increments are not constant but follow a distribution (in
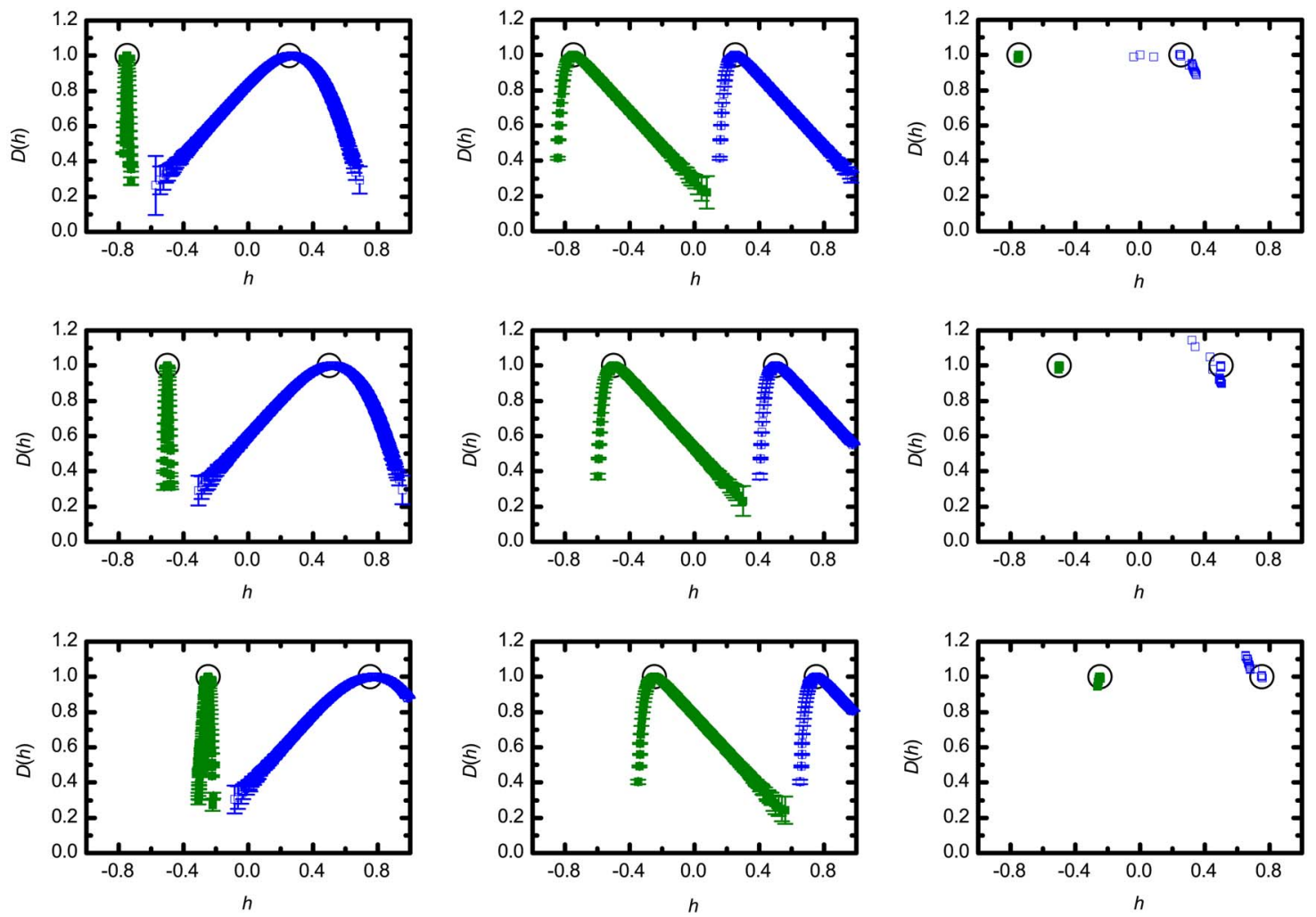

FIG. 6. (Color online) Estimated singularity spectra $D(h)$ computed with the local singularity method (left-hand side), the punctual singularity method (center), and the moment method (right-hand side), for the fBm of $H=1 / 4$ (first row), $H=1 / 2$ (second row), and $H=3 / 4$ (third row). The spectra are estimated through linear increments ( $\square$ ) and multifractal measures ( $\square$ ). The circle indicates the theoretical singularity exponent and Hausdorff dimension. 


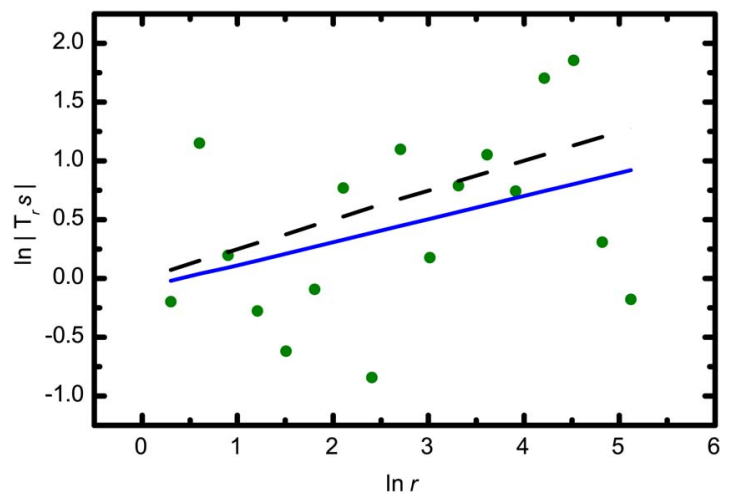

FIG. 7. (Color online) Example of an experimental $\ln \left|\mathbb{T}_{r} s\right|$ vs $\ln r$ plot, used to retrieve the singularity exponent in the local singularity method. $T_{r} s$ is a wavelet extension of the linear increment and the analyzed signal is a $H=1 / 4 \mathrm{fBm}$. The dots correspond to observed data points, which significantly deviate from a straight line. The dashed line is the theoretical regression line, while the continuous line is the actual regression line, corresponding to an exponent $h=0.19$.

fact, they are distributed as the absolute value of a Gaussian variable with dispersion $r^{H}$ ). Any $\mu \mathrm{CF}$ method will reflect this residual random character of the linear increments, so the obtained singularity spectra will always have some dispersion, decreasing with the series size. This dispersion effect becomes strongly evident when linear increments are used for all the methods, with all the spectra peaking at the correct value. Measure functionals provide better results, as the spectra exhibit smaller dispersions than those obtained using linear increment functionals. Notice that measure functionals cannot be defined in the standard way, as the $\mathrm{fBm}$ measure is ill behaved, so the generalized definition presented in Sec. III D has been used.

\section{Local singularity method}

The measure functional gives very nice estimates of deltalike singularity spectra under the application of this method, with the peak exactly on the theoretical singularity exponent $H$. The linear increment functional induces very large dispersed spectra, but also peaking in the right value. Regressions in Eq. (2) with the linear increment functional are found to be of very poor quality, meaning that the linear increment functional is not able to unveil $\mu \mathrm{CF}$ multifractality for this kind of signals. This is not surprising, as the same happens with some real-world multifractal signals [40]. The origin of dispersion is due to numerical limitations, as the asymptotical behavior of Eq. (2) is not numerically achieved with this functional (corrective terms can be important if the functional does not filter them).

This effect can be theoretically studied: As shown in Sec. IV B, the linear increments are Gaussianly distributed, $\Delta_{r} s=N\left(0, r^{2 H}\right)$, so

$$
\ln \left|\Delta_{r} s\right|(t)=H \ln r+\ln |n(t)|,
$$

where $n(t)$ is a $N(0,1)$ random variable. A linear regression of $\ln \left|\Delta_{r} s\right|$ vs $\ln r$ (see Fig. 7) would retrieve the singularity exponent as the slope of the regression line, with an estima-

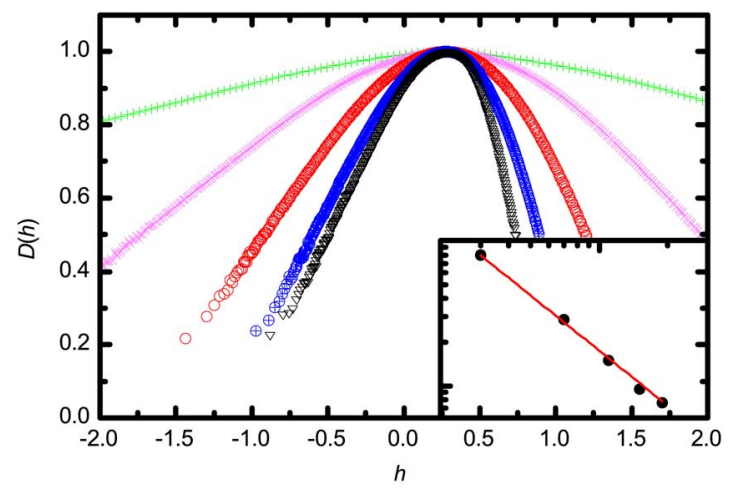

FIG. 8. (Color online) Estimated singularity spectra $D(h)$ computed with the local singularity method, using wavelet projections of linear increments, for a $\mathrm{fBm}$ of $H=1 / 4$. The greatest scale reached is $10^{+1}(+), 10^{+2}(\times), 10^{+3}(\bigcirc), 10^{+4}(\oplus)$, and $10^{+5}(\nabla)$ expressed in units of the resolution scale $r_{0}$. Inset, log-log regression showing that the width at half-height of the spectrum as a function of the logarithm of the scale range, $\mathcal{N}$, approximately decays as a power law of exponent -1.5 , as expected.

tion error depending on the fluctuations of the $\ln |n(t)|$ term. Although the average regression approaches the right exponent-in fact, all of the obtained singularity spectra exactly peak in $H$-the common case is a poor quality regression with a wrongly estimated singularity exponent, leading to the dispersion observed in the singularity spectra. The uncertainty in the regression can be estimated by considering the term $\ln |n(t)|$ as an added noise. Although it is not Gaussian, least squares minimization has been used in the regression, so the uncertainty of the slope derived from least squares will be approximately the dispersion in the singularity exponent. Hence,

$$
\sigma_{H}^{2}=\frac{1}{\mathcal{N}}\left(H^{2}+\frac{12 \sigma_{n^{\prime}}^{2}}{\left(\ln \frac{r_{\max }}{r_{0}}\right)^{2}}\right),
$$

where $n^{\prime}=\ln |n(t)|, \quad \sigma_{n^{\prime}} \approx 1.11$. The number of regression points $\mathcal{N}$ is limited by the scale range $r_{\text {max }} / r_{0}$, as a scale step smaller than 2 supposes that some regression points are identical and uncertainty cannot be reduced. Then, for a fixed scale step, $\mathcal{N} \propto \ln \frac{r_{\max }}{r_{0}}$ and, since $H^{2} \ll 12, \sigma_{H}$ approximately behaves as $\mathcal{N}^{-3 / 2}$. That is, $\sigma_{H}$ is reduced as the scale range grows, but at a very slow pace.

In Fig. 8 we show the spectra of the $H=1 / 4$ ensemble analyzed with different scale ranges. One can see that the obtained spectra slowly converge to the single-point theoretical spectrum as the scale range grows, at the expected rate.

\section{Punctual singularity method}

The obtained spectra have a nicely estimated, deltalike left tail, with the peak in the correct singularity exponent. However, the right tail is linearized instead of having a sharp decay. This problem is very common in this method; it concerns the mode smallness issue discussed in Ref. [13], and has its roots in the limitation to assess very regular behaviors of the signal [that is, the ones associated to large values of 
$h(t)]$ when the method does not take into account the correlation with neighboring points at different scales. Only methods processing information at several scales, as the other two, can accurately resolve the right tail of singularity spectra.

As in the local singularity method, the empirical dispersions in the spectra can be theoretically studied. At the resolution scale, $r_{0}$, both the linear increment and the measure are absolute values of Gaussian variables of dispersion $r_{0}^{H}$ (for the linear increment) and $r_{0}^{H-1}$ (for the measure). Therefore, in application of Eq. (5) we obtain that singularity exponents are distributed as logarithms of Gaussian variables, and the spectra are proportional to the logarithms of these distribution functions. We have observed that these curves exactly coincide with the numerically estimated ones (the singularity spectra shown in Fig. 6), hence the observed dispersion is inherent to the method.

\section{Moment method}

Moments are evaluated over the range $p=-4$ to $p=8$. Due to numerical limitations, the canonical curves $\tau_{p}$ are not perfect straight lines, so a slight dispersion is induced. The spectra obtained from both measure and linear increment functionals are almost pointlike, usually a bit more disperse for the latter. However, the obtained spectra are too flat, which makes the precise localization of the peak more uncertain than in the local singularity method.

\section{CONCLUSIONS}

In this paper, we have discussed on the validity of the microcanonical multifractal approach, $\mu \mathrm{CF}$, to deal with monofractal systems and, in particular, to evaluate the singularity spectrum from discretized synthetic data. $\mu \mathrm{CF}$ is a powerful methodology to deal with multifractal signals, but so far no attempt to generalize it for strictly fractal sets had been attempted. The issue is relevant, as we generally do not a priori know whether a given system is actually multifractal or simply monofractal. In case that $\mu \mathrm{CF}$ methods had not been able to deal with monofractals, this would have enormously restricted their applicability.

Our main conclusion is that appropriate $\mu \mathrm{CF}$ methods can be applied on monofractals, at least over the common examples of monofractal series that we have generated for this study. Results offered by these $\mu \mathrm{CF}$ approaches are of similar quality than the ones obtained with the classical CF approach. This should shift the choice of use to $\mu \mathrm{CF}$ methods, as they offer as a bonus the possibility of knowing the singularity exponents at each point (which is not so relevant in monofractals, but it is of great value on multifractals $[11,33,34])$. In addition, as $\mu \mathrm{CF}$ methods are less demanding in data than CF methods [13], they offer the possibility of estimating the singularity spectrum up to very small fractal dimensions, i.e., the tails of the $D(h)$ curve, that have been often inaccessible with classical CF methodologies. Notice also that CF methods usually require some tuning of parameters (i.e., the range of moments employed in the analysis, in the case of the moment method), which limits the actual range of singularity exponents that they are resolving. In addition, although the performance of $\mathrm{CF}$ methods can be very good in the sense that they retrieve small dispersed singularity spectra, they sometimes lack good localization of the distribution peak. The local singularity method with measure functionals, on the contrary, does not require fine parameter tuning and always provides a reliable estimate of controlled quality and dispersion of the singularity spectrum, with an almost perfect determination of the distribution peak.

When the results of the different $\mu \mathrm{CF}$ methods presented in this work are compared, we see that the one with the best performance is the local singularity method applied to measure functionals. This is not surprising, as previous validation studies on the performance of multifractal methods over general multifractal signals [13] have shown this same better performance for this method: it requires less assumptions on the signal to be analyzed and it offers quality results over moderated size ensemble. In the study presented in this paper, this method always leads to very narrow spectra, which have good correspondence with the deltalike singularity distribution characteristic to monofractals.

The use of both $\mathrm{CF}$ and $\mu \mathrm{CF}$ methods imply some adaptations to the data features when those are of monofractal nature. We have seen that analysis must be restricted to the support of the signal when it is not of total support; in addition, in the case of signals with undefined gradient, the use of multifractal measures implies a redefinition of the measure as the standard notion is not well defined. All these adaptations, however, are simple and general, and can be implemented by default in the methods, as they yield no modification when the data are not affected by the problem concerned with the adaptation. All the discussion on the necessary adaptations to deal with signals of undefined gradient had not been previously discussed in the literature, in spite of the fact that this problem can potentially affect all the methods to a great extent.

\section{ACKNOWLEDGMENTS}

One of the authors (O.P.) was funded by a Ph.D. grant from Generalitat de Catalunya $(\mathrm{GdC})$. One of the authors (A.T.) was supported by a Ramon y Cajal contract from the Spanish Ministry of Education (MEC). The authors acknowledge financial support from MEC, Contract No. BFM200308258, and from GdC, Contract No. 2005SGR00889.

\section{APPENDIX: PROOF ON THE UNCORRECTED SCALING OF THE fBm MEASURE}

In the text, we have mentioned that the scaling in $r$ of the standard, ill-behaved $\mathrm{fBm}$ measure is $\mu_{r} \sim r^{1}$, instead of the expected behavior, $\mu_{r} \sim r^{H}$ which, in fact, is achieved when we use the regularization presented in Sec. III D. We can give a rough argument justifying this scaling, just by considering the $\mathrm{fBm}$ as the limit of a random walk [20],

$$
s(t+\delta) \approx s(t)+a(t) \delta, \quad \delta \rightarrow 0,
$$

where $a(t)$ is a random variable such that 


$$
a(t)= \begin{cases}+a, & p=\frac{1}{2}, \\ -a, & p=\frac{1}{2},\end{cases}
$$

with nontrivial correlations between $a(t)$ and $a\left(t^{\prime}\right)$ at different times $t, t^{\prime}$, but always of the same modulus, $|a(t)|$ $=a \forall t$. In order to recover the correct scaling associated to the $\mathrm{fBm}$, the value of $a$ should be $\delta^{H}$ (see the conditions presented in Sec. IV B). Then,

$$
\mu_{r}^{(\delta)}=\sum_{i=1}^{N}\left|\delta^{H}\right|=N \delta^{H}=r^{1} \delta^{H-1} \sim r^{1},
$$

where we use the same notation as in Sec. III D. Also, we implicitly assume the limits $\delta \rightarrow 0, N \rightarrow \infty$. with $r=N \delta$. (In the following derivations, the same will apply.)

However, this result does not prove that, for a true $\mathrm{fBm}$, the scaling must be the same. Actually, we can give a more rigorous proof of the presented scaling, considering true Gaussian increments and taking into account correlations. First, we define the modulus of the increments around a point $t_{0}$ as

$$
x_{i}=\left|s\left[t_{0}+(i+1) \delta\right]-s\left(t_{0}+i \delta\right)\right| .
$$

Then, the standard measure of $N$ points, noted as $\mu_{r}^{(\delta)}\left(t_{0}\right)$ in Sec. III D, will be

$$
\mu_{N}=x_{1}+\cdots+x_{N}
$$

where now we will use the more simple notation $\mu_{N}$, to set the accent on the number of points. The order $p$ power of this measure can be expressed through the multinomial formula

$$
\mu_{N}^{p}=\sum_{\substack{i_{1}=0 \\ i_{1}+\cdots+i_{N}=p}}^{p} \cdots \sum_{\substack{i_{N}=0 \\ i_{1} ! \cdots i_{N} !}}^{p} x_{1}^{i_{1}} \cdots x_{N}^{i_{N}}
$$

which can also be expressed as a sum of terms containing $p$ (equal or different) factors,

$$
\mu_{N}^{p}=\sum_{i_{1}, \cdots, i_{p}} x_{i_{1}} \cdots x_{i_{p}}
$$

that can be arranged by the multiplicity, namely,

$$
\mu_{N}^{p}=\underbrace{\sum_{i_{1} \neq i_{2} \neq \cdots \neq i_{p}} x_{i_{1}} \cdots x_{i_{p}}}_{N^{p}-N^{p-1}}+\underbrace{\sum_{i_{1} \neq i_{2} \neq \cdots \neq i_{p_{-}}} x_{i_{1}}^{2} \cdots x_{i_{p-1}}}_{N^{p-1}-N^{p-2}}+\ldots+\sum_{i_{1}=1}^{N} x_{i_{1}}^{p}
$$

for $p \leqslant N$. The underbraces indicate the number of equivalent terms (i.e., those that have the same multiplicity) on each sum. Since all the terms are positive, and their contributions do not directly depend on $N$, the first sum will be typically the largest contribution to $\mu_{N}^{p}$ as $N \rightarrow \infty$. The behavior is not clear when $N$ is not so large, as the moments of $\mu_{N}$ with $p>N$ only have terms with, at most, $N$ different factors. However, high order moments of $\mu_{N}$ only affect the tail of its distribution. Also, even if $N$ is not very large, the first sum in Eq. (A8) has the largest contribution, although the terms in the other sums can be greater than in the first sum. In fact, when we average, we obtain that

$$
\left\langle x_{i_{1}} \cdots x_{i_{p}}\right\rangle \leqslant\left\langle x_{i_{1}}^{2} \cdots x_{i_{p-1}}\right\rangle \leqslant \cdots \leqslant\left\langle x_{i_{1}}^{p}\right\rangle,
$$

something that is due to the generalized Jensen's inequality [15].

Let $x$ be a generic name for any increment $x_{i}$, since these increments are all $N\left(0, \delta^{2 H}\right)$ Gaussianly distributed (see Sec. IV B), it follows that

$$
\left\langle x^{m}\right\rangle=\sqrt{\frac{2}{\pi}} \delta^{n H} \int_{0}^{\infty} d x^{\prime} x^{\prime m} e^{-(1 / 2) x^{\prime 2}}=\frac{\Gamma(m)}{\Gamma\left(\frac{m}{2}\right)} 2^{1-(m / 2)} \delta^{n H} .
$$

For large values of $m$, this can be approximated through the Stirling formula as $\left\langle x^{m}\right\rangle \sim 2^{m / 2} \Gamma\left(\frac{m}{2}\right) \delta^{n H}$. Notice that the actual value of $\delta$ is not relevant to compare the relative contributions of the terms in Eq. (A8), since it has the same contribution, $\delta^{p H}$, in all of these terms.

In the case of a classical Brownian motion, $H=1 / 2$, the increments are independent, and hence

$$
\left\langle x_{i_{1}} \cdots x_{i_{p}}\right\rangle=\langle x\rangle^{p}=\left(\frac{2}{\pi}\right)^{p / 2} \delta^{p H} .
$$

This result allows us to compare the contributions of the first and the second sum in Eq. (A8), when averaged. The terms in the second sum are typically a factor $\frac{\pi}{2}$ greater than those in the first sum, but the second sum only has $\left(N^{p-1}-N^{p-2}\right)$ terms instead of $\left(N^{p}-N^{p-1}\right)$, thus rapidly becomes irrelevant as $N$ increases. The terms in the third sum are a factor 2 greater than the terms in the second sum, but also have less contribution. The last sum has $N$ terms $\left\langle x^{p}\right\rangle$ which, in the worst case, $p=N$, will asymptotically contribute as $2^{N / 2} \Gamma\left(\frac{N}{2}\right)$, anyway lesser than the $\left(N^{N}-N^{N-1}\right)\left(\frac{2}{\pi}\right)^{N / 2}$ of the first sum. Then, the leading contribution is that of the first sum, and so the order $p$ moment of the measure can be approximated as

$$
\left\langle\mu_{N}^{p}\right\rangle \approx N^{p}\langle x\rangle^{p}=N^{p}\left\langle\mu_{1}^{p}\right\rangle \quad \forall p \leqslant N .
$$

Therefore, from Eqs. (2) and (15), it follows that the estimated scaling corresponds to a Hölder exponent $h=0$,

$$
\mu_{N} \approx N \mu_{1} \sim r^{1},
$$

where the $\dot{\approx}$ symbol means that both sides approximately have the same distribution.

For the general case of an index $H \mathrm{fBm}$, correlations between the $x_{i}$ factors in the $x_{i_{1}} \cdots x_{i_{p}}$ terms decay very fast, unless $(1-H)$ is very small. Hence, the scaling in $N$ of the measure is similar to that of the $H=1 / 2$ case, Eq. (A13), for $N \gg 1$. (In our work, we have seen that cases with, e.g., $H=0.9$ and $N=50$ have imperceptible deviations from the $H=1 / 2$ case, but we have not deeply studied this effect, as the regularization proposed in Sec. III D works anyway.) Although Eq. (A11) is not true for a general $\mathrm{fBm}$ with $H \neq 1 / 2$, almost all the factors in $x_{i_{1}} \cdots x_{i_{p}}$ are decorrelated and we can approximate 


$$
\left\langle x_{i_{1}} \cdots x_{i_{p}}\right\rangle \approx\langle x\rangle^{p}
$$

provided that $N$ is sufficiently large. This is due to the fact that correlations between two distant increments decay very fast with distance, and then almost all the factors in $x_{i_{1}} \cdots x_{i_{p}}$ are typically very little correlated as, for large $N$, the $x_{i}$ are typically not close to each other. The same argument applies to each term of Eq. (A8) and then a similar discussion to that of the $H=1 / 2$ case leads to Eq. (A13) also valid in general, although it should be remarked that $\mu_{1}$ does depend on $H$, i.e., in all the cases, the measure has the same scaling in $N$, but it does not mean that it has the same distribution. If $N$ is not so large, the same result is still valid for small orders of moments $p$ which, in fact, give the bulk of the $\mu_{N}$ distribution.

The validity of approximation (A14) lays on how fast the correlations of the increments $x_{i}$ decay with distance. To see this, we define two increments of a fBm near the instant $t_{0}$ separate $n$ points as

$$
\begin{aligned}
& b_{1}=s\left(t_{0}+\delta\right)-s\left(t_{0}\right), \\
& b_{2}=s\left[t_{0}+(n+1) \delta\right]-s\left(t_{0}+n \delta\right) .
\end{aligned}
$$

We also define the correlation between these increments as

$$
C=\frac{\left\langle b_{1} b_{2}\right\rangle}{\delta^{2 H}}=\frac{(n+1)^{2 H}+(n-1)^{2 H}-2 n^{2 H}}{2},
$$

where we use that the increments are $N\left(0, \delta^{2 H}\right)$ distributed. Now we define the covariance matrix $\mathrm{C}$ as

$$
\mathrm{C}=\delta^{2 H}\left(\begin{array}{ll}
1 & C \\
C & 1
\end{array}\right) .
$$

This allows us to write the joint multivariant PDF of the increments $b_{1}$ and $b_{2}$,

$$
\begin{aligned}
f\left(b_{1}, b_{2}\right) & =\frac{1}{\sqrt{\operatorname{det}(2 \pi \mathrm{C})}} e^{-(1 / 2) \vec{b}^{*} \mathrm{C}^{-1} \vec{b}} \\
& =\frac{1}{2 \pi \sqrt{1-C^{2}} \delta^{2 H}} e^{-\left(b_{1}^{2}+b_{2}^{2}-2 C b_{1} b_{2}\right) /\left[2\left(1-C^{2}\right) \delta^{2 H}\right]} .
\end{aligned}
$$

The moduli of the increments, i.e., the $x_{i}$, have a correlation

$$
C_{x_{i}, x_{i+n}}=\frac{\left\langle\left|b_{1}\right|\left|b_{2}\right|\right\rangle-\langle|b|\rangle^{2}}{\left\langle|b|^{2}\right\rangle-\langle|b|\rangle^{2}}=\frac{I}{1-\frac{2}{\pi}},
$$

where we have considered $x_{i}=\left|b_{1}\right|$ and $x_{i+n}=\left|b_{2}\right|$. Notice that the origin points $t_{0}$ in their respective definitions do not need to be the same for the $x$ 's as for the $b$ 's, because the correlation does not depend on the subindex $i$, only on the relative distance $n$. The numerator is abbreviated as $I$ and corresponds to the following Gaussian integral:

$$
\begin{aligned}
I= & \frac{1}{2 \pi} \int_{-\infty}^{\infty} d b_{1} \int_{-\infty}^{\infty} d b_{2}\left|b_{1}\right|\left|b_{2}\right| \\
& \times\left(\frac{1}{\sqrt{1-C^{2}}} e^{-\left(b_{1}^{2}+b_{2}^{2}-2 C b_{1} b_{2}\right) /\left(2-2 C^{2}\right)}-e^{-\left(b_{1}^{2}+b_{2}^{2}\right) / 2}\right) .
\end{aligned}
$$

Notice that we have normalized $b_{1}$ and $b_{2}$, to cancel a $\delta^{2 H}$ factor in the numerator and the denominator of Eq. (A19).

We are specially interested in the tail of the correlation function $C_{x_{i}, x_{i+n}}$, that is, the case where $n \gg 1$. We accept that correlations can be important at short distances, but the validity of Eq. (A14) would depend only on how fast the correlations decay, in order to not affect the moments when $N$ is large enough. From Eq. (A16), it follows that $n \gg 1$ implies $C \ll 1$, for any value of $H$ between 0 and 1 . Then, the integral $I$ can be approximated and easily calculated for this case,

$$
\begin{aligned}
I \approx & \frac{1}{2 \pi} \int_{-\infty}^{\infty} d b_{1} \int_{-\infty}^{\infty} d b_{2}\left|b_{1}\right|\left|b_{2}\right| e^{-\frac{b_{1}^{2}+b_{2}^{2}}{2}} \\
& \times(\underbrace{\left(1+\frac{1}{2} C^{2}\right) e^{-\frac{C^{2} b_{1}^{2}+C^{2} b_{2}^{2}+2 C b_{1} b_{2}}{2}}-1}_{A}) .
\end{aligned}
$$

where we have truncated the Taylor expansions on $C$ at second order. The expression $A$ is approximated as

$$
A \approx-C \underbrace{b_{1} b_{2}}_{\text {vanishes }}+\frac{C^{2}}{2}(\underbrace{-b_{1}^{2}-b_{2}^{2}}_{\Rightarrow I_{1}}+\underbrace{b_{1}^{2} b_{2}^{2}}_{\Rightarrow I_{2}}+\underbrace{1}_{\Rightarrow I_{3}}),
$$

where the first order term vanishes for symmetry reasons. That is why we need the second order terms, which give rise to three integrals, $I \approx \frac{C^{2}}{2}\left(I_{1}+I_{2}+I_{3}\right)$, with values

$$
\begin{aligned}
& I_{1}=-2 \frac{1}{2 \pi} \int_{-\infty}^{\infty} d b|b|^{3} e^{-(1 / 2) b^{2}} \int_{-\infty}^{\infty} d b|b| e^{-(1 / 2) b^{2}}=-\frac{8}{\pi}, \\
& I_{2}=\frac{1}{2 \pi}\left(\int_{-\infty}^{\infty} d b|b|^{3} e^{-(1 / 2) b^{2}}\right)^{2}=\frac{8}{\pi} \\
& I_{3}=\frac{1}{2 \pi}\left(\int_{-\infty}^{\infty} d b|b| e^{-(1 / 2) b^{2}}\right)^{2}=\frac{2}{\pi}
\end{aligned}
$$

so the integral $I$ is well approximated as

$$
I \approx \frac{C^{2}}{\pi}
$$

Moreover, the expression of $C$ in Eq. (A16) is itself approximated as

$$
C \approx H(2 H-1) n^{-2(1-H)}
$$

when $n \gg 1$. Therefore, we obtain that

$$
C_{x_{i}, x_{i+n}} \approx \frac{H^{2}(2 H-1)^{2}}{\pi-2} n^{-4(1-H)}
$$

and a fast decay of the correlations is assured $\forall H<1$. 
[1] T. C. Hasley, M. H. Jensen, L. P. Kadanoff, I. Procaccia, and B. I. Shraiman, Phys. Rev. A 33, 1141 (1986).

[2] B. B. Mandelbrot and C. Evertsz, Nature (London) 348, 143 (1990).

[3] J. McWilliams and J. Weiss, Phys. Fluids A 5, 608 (1993).

[4] D. L. Ruderman and W. Bialek, Phys. Rev. Lett. 73, 814 (1994).

[5] Z. S. She and E. Leveque, Phys. Rev. Lett. 72, 336 (1994).

[6] A. Davis, A. Marshak, and W. Wiscombe, in Wavelets in Geophysics, edited by E. Foufoula-Georgiou and P. Kumar (Academic, New York, 1994), pp. 249-298.

[7] R. N. Mantegna and H. E. Stanley, Nature (London) 376, 46 (1995).

[8] B. Duplantier, Phys. Rev. Lett. 84, 1363 (2000).

[9] P. Gopikrishnan, V. Plerou, Y. Liu, L. A. N. Amaral, X. Gabaix, and H. E. Stanley, Physica A 287, 362 (2000).

[10] P. Ivanov, L. Amaral, A. Goldberger, S. Havlin, M. Rosenblum, Z. Struzik, and H. Stanley, Nature (London) 399, 461 (1999).

[11] A. Turiel and N. Parga, Neural Comput. 12, 763 (2000).

[12] A. Turiel and A. del Pozo, 11, 345 (2002).

[13] A. Turiel, C. Pérez-Vicente, and J. Grazzini, J. Comput. Phys. (to be published).

[14] A. B. Chhabra, C. Meneveau, R. V. Jensen, and K. R. Sreenivasan, Phys. Rev. A 40, 5284 (1989).

[15] W. Rudin, Real and Complex Analysis (McGraw-Hill, New York, 1987)

[16] E. A. Novikov, Phys. Rev. E 50, R3303 (1994).

[17] A. Arneodo et al., Europhys. Lett. 34, 411 (1996).

[18] B. Dubrulle, Phys. Rev. Lett. 73, 959 (1994).

[19] G. Parisi and U. Frisch, in Turbulence and Predictability in Geophysical Fluid Dynamics: Proceeding of the International School of Physics E. Fermi, edited by M. Ghil, R. Benzi, and G. Parisi (North-Holland, Amsterdam, 1985), pp. 84-87.

[20] K. Falconer, Fractal Geometry: Mathematical Foundations and Applications (Wiley, Chichester, 1990).

[21] A. Arneodo, F. Argoul, E. Bacry, J. Elezgaray, and J. F. Muzy, Ondelettes, Multifractales et Turbulence (Diderot Editeur, Paris, France, 1995).
[22] R. Benzi, L. Biferale, A. Crisanti, G. Paladin, M. Vergassola, and A. Vulpiani, Physica D 65, 352 (1993).

[23] R. Benzi, S. Ciliberto, C. Baudet, G. R. Chavarria, and C. Tripiccione, Europhys. Lett. 24, 275 (1993).

[24] U. Frisch, Turbulence (Cambridge University Press, Cambridge, MA, 1995).

[25] B. Castaing, J. Phys. II 6, 105 (1996).

[26] J. F. Muzy, E. Bacry, and A. Arneodo, Phys. Rev. Lett. 67, 3515 (1991).

[27] I. Daubechies, Ten Lectures on Wavelets, CBMS-NSF Series in Applied Mathematics (Capital City Press, Montpelier, Vermont, 1992).

[28] S. Mallat, A Wavelet Tour of Signal Processing, 2nd ed. (Academic, New York, 1999).

[29] A. Arneodo, J.-F. Muzy, and D. Sornette, Eur. Phys. J. B 2, 277 (1998).

[30] S. G. Roux, A. Arneodo, and N. Decoster, Eur. Phys. J. B 15, 765 (2000).

[31] P. Kestener and A. Arnéodo, Phys. Rev. Lett. 91, 194501 (2003).

[32] A. Arneodo, in Wavelets: Theory and Applications, edited by G. Erlebacher, M. Y. Hussaini, and L. Jameson, ICASE/LaRC Series in Computational Science and Engineering (Oxford University Press, Oxford, 1996), p. 349.

[33] A. Turiel, G. Mato, N. Parga, and J. P. Nadal, Phys. Rev. Lett. 80, 1098 (1998).

[34] A. Turiel, J. Isern-Fontanet, E. García-Ladona, and J. Font, Phys. Rev. Lett. 95, 104502 (2005).

[35] J. F. Muzy, E. Bacry, and A. Arneodo, Int. J. Bifurcation Chaos Appl. Sci. Eng. 4, 245 (1994).

[36] S. Mallat and W. L. Huang, IEEE Trans. Inf. Theory 38, 617 (1992).

[37] A. Turiel and C. Pérez-Vicente, Physica A 322, 629 (2003).

[38] A. Turiel, J. Grazzini, and H. Yahia, IEEE Trans. Geosci. Remote Sens. Lett. 2, 447 (2005).

[39] A. T. A. Wood and G. Chan, J. Comput. Graph. Stat. 3, 409 (1994).

[40] P. Oswiecimka, J. Kwapien, S. Drozdz, and R. Rak, Acta Phys. Pol. B 36, 2447 (2005). 